\section{Anemia em crianças indígenas da etnia Karapotó}

\section{Anemia in indigenous children of Karapotó ethnic backgrounds}

Janaína Ferro Pereira 1

Maria Alice Araújo Oliveira 2

Juliana Souza Oliveira 3
${ }^{1}$ Universidade Federal de Alagoas. Av. Severino Barbosa, s.n. Bom Sucesso. Campus Arapiraca. Arapiraca, AL, Brasil. CEP: 57.309-005. E-mail: Janainaferro2009@hotmail.com

2 Faculdade de Nutrição. Universidade Federal de Alagoas. Maceió, AL, Brasil.

3 Centro Acadêmico de Vitória. Núcleo Nutrição. Universidade Federal de Pernambuco. Vitória de Santo Antão, PE, Brasil.

\begin{abstract}
Objectives: to identify the prevalence and factors associated with anemia in children of Karapotó ethnic backgrounds.

Methods: a cross-sectional study of hemoglobin dosage levels using a portable Hemocue photometer to collect measurements of weight and height along with socioeconomic data for 99 children between 6 and 59 months of age, all of Karapotó ethnicity. The association between the prevalence of anemia and variables related to children, mothers and families were analyzed, using chi-square tests or Fisher Exact Tests.

Results: the prevalence of anemia in the children was $57.6 \%$, the prevalences of low height for age range, low weight-to-height ratios and low weight for age range in the children were $15.6 \%, 3.0 \%$ and $2.0 \%$ respectively. The occurrence of anemia was associated with younger children, those that spent less studying time with their mother, a large number of family members, those with fewer personal possessions and those that lived outside the group's home area or village ("desaldeados").

Conclusions: the results show that anemia is a serious health problem among Karapotó children, especially the "desaldeados", coinciding with problems of childhood obesity and stunted growth.
\end{abstract}

Key words Anemia, Indians, south american Nutritional status, Child

\section{Resumo}

Objetivos: identificar a prevalência e fatores associados à anemia em crianças indígenas Karapotó.

Métodos: estudo transversal em que foi realizada dosagem de hemoglobina com fotômetro portátil Hemocue, coletadas medidas de peso e estatura e dados socioeconômicos de 99 crianças de 6 a 59 meses da etnia karapotó. Foi analisada a associação entre a prevalência de anemia e variáveis referentes às crianças, às mães e às famílias utilizando teste qui-quadrado ou teste exato de Fisher.

Resultados: a prevalência de anemia nas crianças foi de 57,6\%, as prevalências de baixa estatura para idade, baixo peso para estatura e baixo peso para idade entre as crianças foram de 15,6\%, 3,0\% e 2,0\%, respectivamente. A ocorrência de anemia associou-se à menor idade da criança, a um menor tempo de estudo materno, a um maior número de membros da família, à menor posse de itens de consumo e à moradia fora da aldeia (desaldeados).

Conclusões: os resultados evidenciam que a anemia é um grave problema de saúde entre as crianças Karapotó, principalmente naquelas desaldeadas, coexistindo com problemas como sobrepeso e déficit estatural.

Palavras-chave Anemia, Índios sul-americanos, Estado nutricional, Criança 


\section{Introdução}

A situação de saúde da população indígena no Brasil, mesmo sendo pouco estudada, evidencia condições de marginalização socioeconômica que têm claro impacto sobre o perfil saúde/doença. As doenças infecciosas continuam a ocupar um papel de destaque no perfil epidemiológico indígena, mas já há evidências de que as doenças crônicas não transmissíveis estão acometendo índios em todo o país. A sobreposição de agravos transmissíveis e não transmissíveis pode ocorrer de forma mais intensa e complexa devido à sociodiversidade dessa população. ${ }^{1,2}$

Os povos indígenas do Nordeste brasileiro têm em sua história recente a reconquista de suas terras por meio de lutas e conflitos. Quando assentados, encontram-se em locais diferentes dos de sua origem, com solos empobrecidos e limitados, o que dificulta até a garantia da agricultura de subsistência. Essa confluência de fatores ambientais, socioeconômicos, associados ora à ausência da posse da terra ora a aldeias com infraestrutura sanitária deficiente e por vezes inexistente, tem refletido negativamente no perfil de saúde e nutrição dessas populações, acarretando, a vulnerabilidade a agravos como a anemia, que acometem de forma mais acentuada o grupo infantil. 3

No Estado de Alagoas, a população indígena está distribuída em sete etnias, com um grande contingente populacional de desaldeados, apresentando grande heterogeneidade em sua forma de organização social e institucional, em relação às demandas supridas por órgãos governamentais de assistência aos índios. Os índios aldeados também apresentam problemas desde sua situação territorial até a condição de insegurança alimentar. ${ }^{4}$

Os poucos estudos sobre anemia em populações indígenas no Brasil estão concentrados na Região Norte. Essas populações apresentam taxas mais elevadas de mortalidade infantil, morbimortalidade por doenças infecciosas e parasitárias, desnutrição e anemia que as taxas nacionais. ${ }^{5}$ As prevalências de anemia no grupo de crianças de seis meses a cinco anos, variam de $60 \%$ em crianças Kamaiurá, 6 a $84 \%$ em crianças Suruí. ${ }^{2}$ O $1^{\circ}$ Inquérito Nacional de Saúde e Nutrição dos Povos Indígenas, realizado em 2009,7 considera a anemia como uma das principais endemias entre os povos indígenas no Brasil, estando presente em todas as macrorregiões. $\mathrm{O}$ único estudo realizado em Alagoas identificou no povo Xucuru - Kariri, a prevalência de $48,4 \%$ de anemia em crianças de 0 a 9 anos, porém, como os autores não estratificaram seus resultados por faixa etária, ficou difícil a comparação com outros estudos. ${ }^{8}$

Em 2006, o Distrito Sanitário Especial IndígenaAlagoas/Sergipe da Fundação Nacional de Saúde (DSEI-AL/SE, FUNASA), por ocasião da implantação do Sistema de Vigilância Alimentar e Nutricional (SISVAN) indígena, realizou uma avaliação nutricional de crianças de seis áreas indígenas, entre as quais as da etnia Karapotó, habitantes das Aldeias Plak-Ô e do povoado Terra Nova localizados no município de São Sebastião, Alagoas. Nesses locais a prevalência de risco nutricional e baixo peso para idade foi maior do que nas demais populações indígenas do estado de Alagoas. O presente trabalho insere-se em um projeto de pesquisa intitulada "Diagnóstico alimentar e nutricional: implantação de área sentinela nas aldeias do povo Karapotó - Alagoas". Portanto, tendo em vista a escassez de estudos sobre anemia em populações indígenas, considerou-se oportuno determinar a prevalência de anemia e os fatores associados ao ambiente, socio-econômicos, em crianças indígenas Karapotó, moradoras de duas áreas com reconhecidas dife-renças em relação à posse da terra.

\section{Métodos}

O estudo foi realizado na Aldeia Plak-Ô e povoado Terra Nova (desaldeados), situadas no município de São Sebastião, Alagoas. A Aldeia Plak-Ô está localizada na antiga fazenda Tabuado com 270 ha, adquirida pela Fundação Nacional do Índio (FUNAI), em 2003. Possui uma população estimada de 400 pessoas. O povoado Terra Nova localiza-se na zona rural do município de São Sebastião, onde se encontram integrados ao restante da população local, aproximadamente 502 índios, segundo o censo realizado pela FUNASA em 2003.

Estudo do tipo transversal, inicialmente foi realizado um levantamento censitário junto aos agentes indígenas de saúde para identificar todas as crianças de 6 a 59 meses da etnia Karapotó, totalizando 103 crianças. $\mathrm{O}$ trabalho de campo foi realizado entre os meses de agosto a outubro de 2008 , e a população estudada foi constituída por todas as crianças de 6 a 59 meses da etnia Karapotó presentes nos momentos de coleta de dados $(n=99)$, residentes na Aldeia Plak-Ô $(n=34)$ e povoado Terra Nova $(n=65)$, município de São Sebastião - Alagoas. Foram excluídas do estudo quatro crianças, que não se encontravam na aldeia e povoado nos dias de coleta de dados.

A equipe de trabalho responsável pela coleta de dados foi formada por três docentes da Universidade Federal de Alagoas, e sete alunos que foram 
treinados e devidamente supervisionados durante todo o processo de coleta de dados. Durante esse período, a equipe esteve na aldeia e no povoado por quatro vezes, até completar a coleta de dados prevista. Foram realizadas entrevistas nos domicílios com as mães ou responsáveis, utilizando formulário padronizado e previamente testado em estudo anterior da Faculdade de Nutrição da Universidade Federal de Alagoas, 9,10 com a inclusão de algumas variáveis, entre as quais informações sobre aspectos demográficos, socioeconômicos, de saúde e inserção em programas de transferência de renda. A avaliação antropométrica e a dosagem de hemoglobina foram realizadas nos polos bases da aldeia e do povoado no momento em que aconteciam as visitas domiciliares. Após cada dia de coleta dos dados, os questionários eram revistos pela equipe de campo para avaliar a consistência das informações.

A avaliação antropométrica foi realizada através da aferição das medidas corporais das crianças e de suas mães. A massa corporal foi obtida por meio de balança eletrônica portátil com capacidade para 200 $\mathrm{kg}$ e sensibilidade para $50 \mathrm{~g}$ (Marte LC200PP). As crianças foram pesadas e medidas descalças e com vestimenta mínima. O comprimento dos menores de dois anos foi verificado na posição de decúbito dorsal, utilizando infantômetro com escala de 100 $\mathrm{cm}$ e com resolução de $0,1 \mathrm{~cm}$. As crianças maiores de dois anos foram medidas em posição ortostática em um estadiômetro vertical, com $213 \mathrm{~cm}$. Todas as medidas de comprimento foram verificadas duas vezes e, ao ocorrer discrepância superior a $0,5 \mathrm{~cm}$ entre as duas medidas, uma terceira era realizada, determinando, assim, a média das mensurações.

Para classificar o estado nutricional das crianças foram utilizados os índices peso/idade (P/I), peso/estatura $(\mathrm{P} / \mathrm{E})$ e estatura/idade $(\mathrm{E} / \mathrm{I})$, expressos em valores de escore $\mathrm{Z}$ e comparados aos dados de referência antropométrica da Organização Mundial da Saúde WHO ANTHRO, 2007.11 As medidas maternas foram utilizadas para compor o índice de massa corporal (IMC) com pontos de corte definidos pela World Health Organization, ${ }^{12}$ baixo peso $(<18,5)$, eutrófica $(<18,5$ e $<25)$ e sobrepeso, obesidade $(\geq 25$ e $<30) .{ }^{2}$ Para fins de análise, optouse por agrupá-los em duas categorias $(<25$ e $\geq 25)$. Foram excluídas dessa análise todas as mães gestantes.

A hemoglobina $(\mathrm{Hb})$ foi dosada nas mães e crianças de 6 a 59 meses mediante o uso de um fotômetro portátil Hemocue, seguindo procedimento padrão com coleta de gota de sangue por punção de polpa digital da criança e de sua mãe. O ponto de corte adotado para diagnóstico da anemia foi de valor de hemoglobina $<11,0 \mathrm{~g} / \mathrm{dL}$ nas crianças, nas mães não grávidas $<12,0 \mathrm{~g} / \mathrm{dL}$. Para caracterizar a gravidade do quadro, utilizou-se a seguinte classificação: anemia leve $(9,0<\mathrm{HB}<11,0)$, anemia moderada $(7,0 \leq \mathrm{HB} \leq 9,0)$ e anemia grave $(\mathrm{HB}<7,0) .13$

No processamento dos dados foi utilizado o programa Epi-info, versão 6.04, em dupla entrada para garantir a qualidade e consistência das informações. A validação foi realizada através do módulo Validate. A análise dos dados foi realizada através do programa Statistical Package for Social Sciences (SPSS), versão 12.0 for Windows.

Os dados foram categorizadas para a montagem de tabelas de contingência com associações estatísticas entre duas variáveis aleatórias. Para comparar frequências, aplicou-se o teste qui-quadrado ou o teste exato de Fisher. Para todas as análises, foi consi-derado o nível de significância de $p<0,05$.

$\mathrm{O}$ estudo foi aprovado pelo Comitê de Ética em Pesquisa (CEP) da Universidade Federal de Alagoas (processo $\mathrm{n}^{\circ} 006828 / 2008-88$ ). Todas as crianças tiveram permissão para participar do estudo mediante assinatura de um termo de consentimento livre e esclarecido pelos pais ou responsáveis legais. Foi assegurada a todos os participantes a confidencialidade das informações. A todas as crianças e mães diagnosticadas anêmicas, foi garantida, com o apoio do Distrito Especial Indígena (Dsei/AL), a suplementação de ferro.

\section{Resultados}

A prevalência de anemia entre as crianças foi de $57,6 \%$ sendo a maioria classificada como leve e moderada. Foram encontradas associações significativas entre a anemia e a idade da criança, mas não foi encontrada esta associação entre anemia e o sexo da criança. Entre as mães, 41,6\% encontravam-se anêmicas. As prevalências de baixa estatura para idade, baixo peso para estatura e baixo peso para idade entre as crianças foram de 15,6\%, 3,0\% e $2,0 \%$, respectivamente. Considerando o índice de massa corporal (IMC) materno, detectou-se que $46,4 \%$ encontravam-se com excesso de peso (Tabela $1)$.

Quanto à escolaridade, observou-se que a maioria das mães apresentava menos de cinco anos de estudo $(57,5 \%)$ e que a prevalência de crianças anêmicas foi significativamente maior nesse grupo (Tabela 2 ).

Em relação à situação socioeconômica, a maioria das famílias $(90,5 \%)$ vivia com menos de $1 / 4$ de salário mínimo per capita mensal e mais da metade 
estava inserida em programa de transferência de renda governamental (Tabela 1). A maioria das famílias não trata sua água de consumo. Não houve diferenças estatisticamente significativas entre prevalência de anemia, categorias de renda e número de cômodos da casa. O maior número de membros da família e menor posse de itens de consumo apresentaram associação estatisticamente significativa com a maior prevalência de anemia (Tabela 2).

Houve associação entre a prevalência de anemia e o local de moradia, sendo que a maioria das crianças anêmicas encontrava-se entre as famílias desaldeadas que viviam no povoado Terra Nova (Tabela 2).

Quanto à avaliação antropométrica das crianças, não houve associação entre estado nutricional e anemia (Tabela 3).

A maioria das crianças foi desmamada após um ano $(65,0 \%)$. Não houve associação de anemia com a idade do desmame, bem como com a suplementação de vitamina $\mathrm{A}$ e ferro nos últimos seis meses. Vale ressaltar que apenas 3,5\% das crianças faziam uso de sulfato ferroso (Tabela 3).

Tabela 1

Caracterização da distribuição de anemia, estado nutricional e indicadores socioeconômicos nas crianças de 6 a 59 meses, mães e famílias indígenas Karapotó. São Sebastião, Alagoas, Brasil, 2008.

\begin{tabular}{|c|c|c|}
\hline Variáveis & $\mathbf{N}$ & $\%$ \\
\hline \multicolumn{3}{|l|}{ Anemia nas crianças } \\
\hline Não anêmicos & 42 & 42,4 \\
\hline Anêmicos & 57 & 57,6 \\
\hline Anemia leve & 39 & 39,4 \\
\hline Anemia moderada & 16 & 16,2 \\
\hline Anemia grave & 2 & 2,0 \\
\hline \multicolumn{3}{|l|}{ Estatura para idade da criança } \\
\hline Desnutrição (Z<-2 DP) & 15 & 15,6 \\
\hline Risco nutricional ( $\geq-2$ e $<-1$ DP) & 28 & 29,2 \\
\hline Eutrofia $(\geq-1$ e $<1 \mathrm{DP})$ & 53 & 55,2 \\
\hline \multicolumn{3}{|l|}{ Peso para estatura da criança } \\
\hline Desnutrição (Z<-2 DP) & 3 & 3,0 \\
\hline Risco nutricional ( $\geq-2$ e $<-1$ DP) & 4 & 4,2 \\
\hline Eutrofia ( $\geq-1$ e $<1$ DP) & 67 & 69,0 \\
\hline Risco de sobrepeso ( $\geq 1$ e $<2$ DP) & 17 & 17,6 \\
\hline Sobrepeso ( 22 DP) & 6 & 6,2 \\
\hline \multicolumn{3}{|l|}{ Peso para idade da criança } \\
\hline Desnutrição (Z<-2 DP) & 2 & 2,0 \\
\hline Risco nutricional ( $\geq-2$ e $<-1$ DP) & 20 & 20,4 \\
\hline Eutrofia $(\geq-1$ e $<1 \mathrm{DP})$ & 62 & 63,3 \\
\hline Risco de sobrepeso ( $\geq 1$ e $<2$ DP) & 12 & 12,3 \\
\hline Sobrepeso( $\geq 2$ DP) & 2 & 2,0 \\
\hline \multicolumn{3}{|l|}{ Anemia materna } \\
\hline Não anêmicas & 49 & 58,4 \\
\hline Anêmicas & 35 & 41,6 \\
\hline \multicolumn{3}{|l|}{ Índice de massa corporal materno } \\
\hline$<18,5 \mathrm{~kg} / \mathrm{m}^{2}-$ Déficit & 2 & 2,4 \\
\hline 18,5 a $24,9 \mathrm{~kg} / \mathrm{m}^{2}$ - Eutrofia & 43 & 51,2 \\
\hline 25 a $29,9 \mathrm{Kg} / \mathrm{m}^{2}$ - Sobrepeso & 25 & 29,8 \\
\hline$\geq 30 \mathrm{~kg} / \mathrm{m}^{2}$ - Obesidade & 14 & 16,6 \\
\hline \multicolumn{3}{|c|}{ Renda per capita familiar (salário mínimo) } \\
\hline$<0,25$ & 77 & 90,5 \\
\hline$\geq 0,25$ & 8 & 9,5 \\
\hline \multicolumn{3}{|c|}{ Programa de transferência de renda nas famílias } \\
\hline $\operatorname{Sim}$ & 49 & 57,6 \\
\hline Não & 36 & 42,4 \\
\hline
\end{tabular}

Salário mínimo $=\mathrm{R} \$ 415,00$. 
Distribuição da concentração de hemoglobina e prevalência de anemia segundo sexo, idade, características maternas, indicadores socioeconômicos e localidade de moradia das crianças indígenas Karapotó de 6 a 59 meses de idade. São Sebastião, Alagoas, Brasil, 2008.

\begin{tabular}{|c|c|c|c|c|c|}
\hline \multirow[t]{2}{*}{ Variáveis } & \multirow[t]{2}{*}{ N } & \multirow[t]{2}{*}{$\%$} & \multicolumn{2}{|c|}{ Anemia* } & \multirow[t]{2}{*}{$p^{* *}$} \\
\hline & & & $\mathrm{n}$ & $\%$ & \\
\hline \multicolumn{6}{|c|}{ Idade das crianças (meses) } \\
\hline $06-24$ & 33 & 35,2 & 24 & 72,7 & 0,028 \\
\hline $25-59$ & 61 & 64,8 & 30 & 49,1 & \\
\hline \multicolumn{6}{|l|}{ Sexo } \\
\hline Feminino & 46 & 46,6 & 26 & 56,5 & 0,84 \\
\hline Masculino & 53 & 53,4 & 31 & 58,4 & \\
\hline \multicolumn{6}{|c|}{ Idade materna (anos) } \\
\hline$<30$ & 49 & 52,2 & 27 & 55,1 & 0,68 \\
\hline$\geq 30$ & 45 & 47,8 & 27 & 60,0 & \\
\hline \multicolumn{6}{|c|}{ Anemia na mãe } \\
\hline $\operatorname{sim}$ & 39 & 39,4 & 25 & 64,1 & 0,30 \\
\hline Não & 60 & 60,6 & 32 & 53,3 & \\
\hline \multicolumn{6}{|c|}{ Escolaridade materna } \\
\hline \multicolumn{6}{|c|}{ Mãe lê e escreve } \\
\hline Não & 32 & 33,4 & 22 & 68,7 & 0,34 \\
\hline Sim & 64 & 66,6 & 32 & 50,0 & \\
\hline \multicolumn{6}{|c|}{$\mathrm{N}^{\circ}$ de anos de estudo materno } \\
\hline$\leq 5$ & 42 & 57,5 & 26 & 61,9 & 0,02 \\
\hline$>5$ & 31 & 42,5 & 11 & 35,4 & \\
\hline \multicolumn{6}{|c|}{ Número de membros da família } \\
\hline$\leq 5$ & 71 & 71,8 & 36 & 50,7 & 0,02 \\
\hline$>5$ & 28 & 28,2 & 21 & 75,0 & \\
\hline \multicolumn{6}{|c|}{ Renda per capita (salário mínimo) } \\
\hline$<0,25$ & 70 & 71,4 & 42 & 60,0 & 0,34 \\
\hline$>0,26$ & 28 & 28,6 & 15 & 53,5 & \\
\hline \multicolumn{6}{|c|}{ Número de cômodos da casa } \\
\hline$\leq 5$ & 47 & 47,4 & 25 & 53,1 & 0,40 \\
\hline$>5$ & 52 & 52,6 & 32 & 61,5 & \\
\hline \multicolumn{6}{|c|}{ Itens de consumo } \\
\hline$\leq 5$ & 44 & 44,4 & 31 & 70,4 & 0,02 \\
\hline$>5$ & 55 & 55,6 & 26 & 42,2 & \\
\hline \multicolumn{6}{|c|}{ Tratamento de água de consumo } \\
\hline $\operatorname{sim}$ & 22 & 22,3 & 11 & 50,0 & 0,41 \\
\hline Não & 77 & 77,7 & 46 & 59,7 & \\
\hline \multicolumn{6}{|c|}{ Inscrição no bolsa família } \\
\hline Não & 42 & 42,4 & 22 & 52,3 & 0,36 \\
\hline $\operatorname{sim}$ & 57 & 57,6 & 35 & 61,4 & \\
\hline \multicolumn{6}{|l|}{ Aldeia } \\
\hline Terra Nova & 65 & 65,6 & 46 & 70,7 & 0,001 \\
\hline Plak-Ô & 34 & 34,4 & 11 & 32,3 & \\
\hline
\end{tabular}

\footnotetext{
* Anemia $=$ hemoglobina $<11 \mathrm{~g} / \mathrm{dL} ; * *$ diferenças pelo teste $\chi^{2}$.
} 
Prevalência de anemia segundo estado nutricional e condições de saúde, das crianças indígenas Karapotó de 6 a 59 meses e de suas respectivas mães. São Sebastião, Alagoas, Brasil, 2008.

\begin{tabular}{|c|c|c|c|c|c|}
\hline \multirow[t]{2}{*}{ Variáveis } & \multirow[t]{2}{*}{$\mathbf{N}$} & \multirow[t]{2}{*}{$\%$} & \multicolumn{2}{|c|}{ Anemia* } & \multirow[t]{2}{*}{$p^{* *}$} \\
\hline & & & $\mathrm{n}$ & $\%$ & \\
\hline \multicolumn{6}{|c|}{ Estatura para idade da criança } \\
\hline 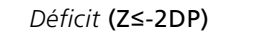 & 15 & 15,7 & 12 & 80,0 & 0,086 \\
\hline 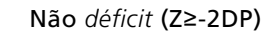 & 81 & 84,3 & 43 & 53,0 & \\
\hline \multicolumn{6}{|c|}{ Peso para idade da criança } \\
\hline Déficit (Z<-2DP) & 2 & 2,0 & 2 & 100,0 & 0,50 \\
\hline 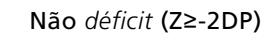 & 96 & 98,0 & 54 & 57,4 & \\
\hline \multicolumn{6}{|c|}{ Peso para estatura da criança } \\
\hline Déficit (Z<-2DP) & 3 & 3,0 & 3 & 100,0 & 0,26 \\
\hline Não déficit (ZZ-2DP) & 94 & 97,0 & 53 & 56,3 & \\
\hline \multicolumn{6}{|c|}{ Índice de massa corporal materno } \\
\hline$<25 \mathrm{~kg} / \mathrm{m}^{2}$ & 54 & 60,0 & 31 & 57,4 & 1,00 \\
\hline$\geq 25 \mathrm{~kg} / \mathrm{m}^{2}$ & 36 & 40,0 & 20 & 55,5 & \\
\hline \multicolumn{6}{|c|}{$\begin{array}{l}\text { Criança recebeu vitamina A } \\
\text { (nos últimos seis meses) }\end{array}$} \\
\hline Sim & 67 & 76,1 & 38 & 56,7 & 0,45 \\
\hline Não & 21 & 23,8 & 14 & 66,6 & \\
\hline \multicolumn{6}{|c|}{$\begin{array}{l}\text { Criança fez uso de sulfato ferroso } \\
\text { (nos últimos seis meses) }\end{array}$} \\
\hline Sim & 13 & 3,5 & 6 & 46,1 & 0,54 \\
\hline Não & 83 & 86,4 & 49 & 59,0 & \\
\hline \multicolumn{6}{|c|}{ Idade de desmame da criança (meses) } \\
\hline$\leq 12$ & 41 & 65,0 & 25 & 60,9 & 0,10 \\
\hline$>12$ & 22 & 22,9 & 9 & 40,9 & \\
\hline
\end{tabular}

* Anemia $=$ hemoglobina $<11 \mathrm{~g} / \mathrm{dL} ; * *$ diferenças pelo teste $\chi^{2}$.

\section{Discussão}

A prevalência de anemia no grupo estudado $(57,6 \%)$ é comparável às prevalências encontradas em outras populações indígenas como os Kamaiurá $(60,0 \%)^{6} \mathrm{e}$ Térenas $(52,0 \%),{ }^{1}$ nessa mesma faixa etária. Elevados percentuais também foram encontrados em outros estudos com crianças índias e não índias como observaram alguns autores no país. 2,9,10,14-16 Porém, segundo Khambalia et al., 17 o problema da anemia é muito maior entre os grupos indígenas.

Em relação à idade, o grupo de crianças de 6 a 24 meses foi o mais atingido pela anemia, estando de acordo com outros estudos realizados no país com populações indígenas. ${ }^{1,2}$ Isto ocorre devido às necessidades elevadas de ferro frente à maior velocidade de crescimento nessa faixa etária, desmame precoce e alimentação complementar pobre em ferro ou de baixa biodisponibilidade. 1,2

A situação materna só reafirma as condições das crianças, ressaltando-se que os resultados aqui expostos não representam todo o grupo de mulheres Karapotó em idade fértil, mas servem de indicativo para o problema que também representa a anemia nesse grupo (41,6\%). Os dados encontrados estão em consonância com os resultados do Inquérito Nacional de Saúde e Nutrição dos Povos Indígenas $(2009)^{7}$ que chamam atenção para níveis de anemia nas mulheres indígenas muito superiores aos que vêm sendo descritos em pesquisas para a população brasileira em geral. Estudo de Orellana et al.,18 reafirma a grave situação com prevalência de anemia de $67,3 \%$ em mulheres indígenas Suruí, resultado superiores ao encontrado nos Karapotós.

Assim como em outros estudos no Mundo e no Brasil,19,20 a anemia está associada a precárias condições de vida, ${ }^{17}$ habitação e maior número de moradores na residência, o que, segundo Netto et $a l ., 21$ favorece uma menor disponibilidade alimentar pela divisão da comida. A menor posse de itens de 
consumo também se relaciona à ocorrência de anemia, refletindo na baixa condição econômica das famílias avaliadas. Diversos autores justificam a influência da escolaridade materna sobre o estado nutricional das crianças, tendo em vista seu efeito sobre a renda familiar que favorece a maior ou menor disponibilidade quantitativa e qualitativa alimentar e a melhoria nos cuidados com sua saúde e a da criança. ${ }^{9,19,20}$ Porém não encontramos nesse estudo associação entre renda e anemia, isso possivelmente em virtude da homogeneidade da situação socioeconômica precária entre os Karapotó e de seu modo de vida, caracterizado pela produção agrícola de subsistência e sem fins lucrativos.

A associação detectada entre a ocorrência de anemia e o local de moradia reflete que a saúde dos povos indígenas pode estar intimamente ligada à posse e ocupação das terras. $22 \mathrm{Na}$ aldeia Plak-Ô, a garantia da posse e utilização da terra demonstra uma situação mais favorável em relação à dos índios moradores do povoado Terra Nova, que vivem misturados a não índios e sem uma situação definida em relação à posse de sua terra.

Os resultados encontrados neste estudo sobre o estado nutricional das crianças corroboram os encontrados em outros estudos indígenas no Brasil,1,6 com o predomínio de déficit estatural, o que reflete um quadro de desnutrição crônica nesse grupo infantil. De modo geral, estudos de tendência secular no mundo e em várias regiões do Brasil têm mostrado um aumento da estatura, ${ }^{23}$ porém em crianças indígenas do alto Xingu, em 12 anos de acompanhamento, não foi detectada melhora no déficit estatural nesse período, 1 o que demonstra a importância do monitoramento do estado nutricional nesses grupos.

A suplementação de vitamina A está implantada nas áreas indígenas de Alagoas desde abril de 2007, o Ministério da Saúde 24 preconiza como meta para cobertura pelo Programa Nacional de Suplementação de Vitamina A, 100\% na primeira dose, para crianças de 6 a 59 meses de idade. Nos Karapotós,

\section{Referências}

1. Morais MB, Alves GMS, Fagundes-Neto U. Estado nutricional de crianças índias terenas: evolução do peso e estatura e prevalência atual de anemia. J Pediatr. 2005; 81 : 383-9.

2. Orellana JDY, Coimbra CEA, Lourenço AEP. Estado nutricional e anemia em crianças Suruí, Amazônia, Brasil. J Pediatr. 2006; 82: 383-8.

3. Leite MS. Nutrição e alimentação em saúde indígena: notas sobre a importância e a situação atual. In: Garnelo L, Pontes
$76,1 \%$ das crianças receberam uma dose deste nutriente nos últimos seis meses. O mesmo não acontece com o Programa de Suplementação de Ferro que no momento da coleta de dados ainda não havia sido implantando pelo DSEI/AL-SE e as poucas crianças $(3,5 \%)$ que fizeram uso de sulfato ferroso, o fizeram para tratamento e não de forma preventiva conforme preconizado pelo programa. Não foi encontrada associação entre anemia e o uso de vitamina A e sulfato ferroso.

Apesar de o inquérito dietético ter sido contemplado no projeto original, para o presente trabalho não foram analisados fatores dietéticos relacionados à alimentação das crianças, constituindo, assim, uma limitação na discussão dos determinantes da anemia no grupo estudado.

É importante reafirmar que a anemia configurase numa complexa rede de fatores que tem determinação múltipla, envolvendo aspectos biológicos, demográficos, socioeconômicos e culturais, 25 portanto seu controle também se torna complexo e deve enfocar várias medidas, garantindo, assim, maior eficácia nos resultados. A identificação desse agravo e dos fatores a ele associados é essencial para o planejamento de estratégias adequadas a seu combate. 26

O enfrentamento da anemia em termos populacionais ultrapassa as ações do setor saúde, uma vez que vai de encontro ao desenvolvimento desigual e à precariedade das condições de vida a que estão submetidos os índios no nordeste brasileiro. No entanto, a curto e médio prazo, ações direcionadas para a garantia do direito à alimentação adequada e à saúde devem ser asseguradas. É imprescindível que haja uma assistência pré-natal adequada para o controle da anemia na gestação, com ações educativas voltadas para a promoção da alimentação saudável e incentivo ao aleitamento materno, intensificação das ações de vigilância alimentar e nutricional, implantação e/ou valorização do Programa de Suplementação de Ferro, entre outras ações.

AL, orgs. Saúde Indígena: uma introdução ao tema. Brasília: MEC-SECADI; 2012. p. 157.

4. Almeida LS. Dois dedos de prosa com os Karapotó. 2 ed. Maceió: Edufal; 1998.

5. Mondini L, Rodrigues DA, Gimeno SGA, Baruzzi RG. Estado nutricional e níveis de hemoglobina em crianças Aruak e Karibe - povos indígenas do Alto Xingu, Brasil Central, 2001-2002. Rev Bras Epidemiol. 2009; 12: 46977. 
6. Mondini L, Canó EM, Fagundes U, Lima EES, Rodrigues D, Baruzzi RG. Condições de nutrição em crianças Kamaiurá: povo indígena do Alto Xingu, Brasil Central. Rev Bras Epidemiol. 2007; 10: 39-47.

7. ABRASCO (Associação Brasileira de Pós-Graduação em Saúde Coletiva). Inquérito Nacional de Saúde e Nutrição dos Povos Indígenas: Relatório Final. Rio de Janeiro, RJ; 2009.

8. Almeida LS, Vilela R, Passos F. Xucuru -Kariri: saúde na fazenda - Canto. Maceió: Edufal; 2005.

9. Ferreira SH, Assunção ML, Vasconcelos VS, Melo FP, Oliveira CG, Santos TO. Saúde de populações marginalizadas: desnutrição, anemia e enteroparasitoses em crianças de uma favela do "movimento sem teto", Maceió, Alagoas. Rev Bras Saúde Matern Infant. 2002; 2: 177-85.

10. Santos CD, Santos LMP, Figueirôa JN, Marroquim PMG Oliveira MAA. Anemia em escolares da primeira série do ensino fundamental da rede pública de Maceió, Alagoas, Brasil. Cad Saúde Pública. 2002; 18: 1757-63.

11. WHO (World Health Organization). Anthro for personal computeres, version 2, 2007: software for assessing growth and development of the word's children. Geneva; 2007.

12. Word Health Organization/ United Nacinal Children's Fund/United Nation University. Iron deficiency anaemia. Assessment, prevention and control. A guide for programme Managers. Geneva; 2001.

13. Maeyer EM, Dallman P, Gorney JM, Hallberg L, Sood SK, Srkantia SG. Preventing and crontroling iron deficiency anaemia through promary heath care. Geneva: Word Health Organization; 1989.

14. Lima ACVMS, Lira PIC, Romani SAM, Erickmann SH Lima MC. Fatores determinantes dos níveis de hemoglobina em crianças aos 12 meses de vida na zona da mata meridional de Pernambuco. Rev Bras Saúde Matern Infant. 2004; 4: 35-43.

15. Miranda AS, Franceschini SCC, Priore SE, Euclydes MP, Araújo RMM, Ribeiro SMR, Netto MP, Fonseca MM, Rocha DS, Silva DG, Lima NMM, Maffia UCC. Anemia ferropriva e estado nutricional de crianças com idade de 12 a 60 meses do município de Viçosa, MG. Rev Nutr. 2003; 16: $163-9$

16. Martins MC, Santos LM, Lima A, Assis A, Amorim D. Perfil nutricional: desnutrição e anemia no Estado de Sergipe. In: V Congresso da Sociedade Brasileira de Alimentação e Nutrição. 1999. São Paulo: Anais; 1999. p. 184

Recebido em 16 de junho de 2011

Versão final apresentada em 27 de julho de 2012

Aprovado em 21 de setembro de 2012
17. Khambalia AZ, Aimone AM, Stanley H. Burden of anemia among indigenous populations nutrition. Nutr Rev. 2011; 69: 693-719.

18. Orellana J DY, Cunha GM, Santos RV, Coimbra Jr. CEA, Leite MS. Prevalência e fatores associados à anemia em mulheres indígenas Suruí com idade entre 15 e 49 anos, Amazônia, Brasil. Rev Bras Saúde Matern Infant. 2011; 11: 153-61.

19. Lourenço AEP. Avaliação do estado nutricional em relação a aspectos socioeconômicos de adultos indígenas Suruí, Rondônia, Brasil [dissertação]. Rio de Janeiro: Escola Nacional de Saúde Pública da Fundação Oswaldo Cruz; 2006.

20. Monteiro CA, Szarfarc SC, Mondini L. Tendência Secular da anemia na infância na cidade de São Paulo (1984-1996). Rev Saúde Pública. 2000; 34 (Supl.): 62-72.

21. Netto MP, Priore SE, Sant'Ana HMP, Peluzio MCG, Sabarense CM, Silva DG, Franceschini SCC. Prevalência e fatores associados à anemia e deficiência de ferro em crianças de 18 a 24 meses. Arch Latinoam Nutr. 2006; 56: 229-36.

22. Machado C. A terra é nossa vida. Rads. 2004; $22: 8$-11.

23. Capelli JCS, Koifmam S. Avaliação do estado nutricional da comunidade indígena Parkatêjê, Bom Jesus de Tocantins, Pará, Brasil. Cad Saúde Pública. 2001; 17: 4337.

24. Brasil. Ministério da Saúde. Secretaria de Atenção à Saúde. Departamento de Atenção Básica.Vitamina A Mais: Programa Nacional de Suplementação de Vitamina A: Condutas Gerais / Ministério da Saúde,Secretaria de Atenção à Saúde, Departamento de Atenção Básica. Brasília, DF; 2004.

25. Pereira RC, Ferreira LOC, Diniz AS, Batista Filho M, Figueirôa JN. Eficácia da suplementação de ferro associado ou não à vitamina A no controle da anemia em escolares. Cad Saúde Pública. 2007; 23: 1415-21.

26. WHO (World Health Organization). Wordwide prevalence of anaemia 1993-2005: WHO Global database on anaemia. Atlanta; 2008. 